Please quote as: Blohm, I.; Leimeister, J. M. \& Zogaj, S. (2014): Crowdsourcing und Crowd Work - ein Zukunftsmodell der IT-gestützten Arbeitsorganisation?. In: Wirtschaftsinformatik in Wissenschaft und Praxis. Business Engineering. Aufl./Vol.. Hrsg./Editors: Brenner, W. \& Hess, T. Verlag/Publisher: Springer-Verlag, Berlin Heidelberg. Erscheinungsjahr/Year: 2014. Seiten/Pages: 51-64. 


\title{
Crowdsourcing und Crowd Work - ein Zukunftsmodell der IT-gestützten Arbeitsorganisation?
}

\author{
Blohm, Ivo, Universität St. Gallen, Institut für Wirtschaftsinformatik, Müller- \\ Friedberg-Strasse 8, CH-9000 St. Gallen, ivo.blohm@unisg.ch \\ Leimeister, Jan Marco, Universität St. Gallen, Institut für Wirtschaftsinformatik, \\ Müller-Friedberg-Strasse 8, CH-9000 St. Gallen, janmarco.Leimeister@unisg.ch \\ Zogaj, Shkodran, Universität Kassel, Lehrstuhl für Wirtschaftsinformatik, \\ Pfannkuchstraße 1, D-34121 Kassel, zogaj@uni-kassel.de
}

\section{Wertschöpfung und Arbeit im Lichte der Digitalisierung}

Das Internet und die damit verbundenen Möglichkeiten haben jegliche Bereiche der Gesellschaft verändert und nachhaltig geprägt. In nahezu allen Bereichen sind digitale Technologien Auslöser und Begleiter neuer Entwicklungen und teilweise radikaler Veränderungen sowohl auf gesamtgesellschaftlicher als auch auf individueller Ebene. Sie haben Einfluss auf unsere Lebens- und Nutzungsgewohnheiten, bieten Lösungen für aktuelle Probleme und eröffnen neue Möglichkeiten (Bundesministerium für Bildung und Forschung, 2013). Es lässt sich nicht abstreiten, dass das Internet und sonstige digitale Technologien die Art der Arbeit grundlegend verändert haben. Am deutlichsten wird dies bei den sogenannten Digital Natives. Die jüngere Generation in Industrieländern wächst von Geburt an mit digitalen Technologien und dem Internet auf - entsprechend anders sind deren Arbeits- und Kommunikationsformen. Leben und Arbeiten sind ohne Digitalisierung nicht vorstellbar und neue Arbeitsformen haben alte ersetzt (Unterberg, 2010).

Die voranschreitende Digitalisierung hat auch (oder insbesondere) weitreichende Implikationen für Unternehmen und die Art, wie diese ihre Leistungserstellungsprozesse koordinieren und erbringen, insbesondere im Bereich wissensintensiver Arbeit. Die immer noch zunehmende Vernetzung ermöglicht hierbei, Arbeit nach anderen Arbeitsorganisationsprinzipien orts- und zeitunabhängig zu verteilen. Über das Netz wird auf ein großes Reservoir an Arbeitskräften schnell und gezielt zugegriffen. Es werden Aufgaben an eine große Menge an Individuen - der so genannten Crowd (dt. „Menge“; sinngemäß „Menge an Menschen“) verteilt - deren Mitglieder die einzelnen Tätigkeiten über ihre persönlichen Rechner auch asynchron und dezentral erledigen können. Hierdurch können Informationen, Ideen und Lösungen von Menschen aus der ganzen Welt mit geringem Aufwand aggregiert und im Leistungserstellungsprozess integriert werden. Dieses Konzept wird in der Literatur als Crowdsourcing bezeichnet und beschreibt im Allgemeinen die Auslagerung von Unternehmensaufgaben auf eine unabhängige Masse von Menschen über das Internet (Howe, 2006). Die 
Mitglieder einer Crowd agieren hierbei als Digital Worker bzw. Crowd Worker und übernehmen kollektiv Aufgaben, die typischerweise von den Mitarbeitern innerhalb eines Unternehmens vollzogen werden. Crowdsourcing ermöglicht als neue Form der Wertschöpfung beeindruckende Ergebnisse. Sie reichen von sehr schnellen Leistungen (bspw. das Übersetzen eines komplexen Texts in wenigen Stunden), bisher nie gekannten Leistungen (bspw. die Kartographie von Planeten, die Entwicklung von Software und Systemen oder die Schaffung von Wissensbasen wie Wikipedia) bis hin zu Lösungen für gesellschaftlich relevante Fragestellungen (bspw. Crowdsourcing Aktivitäten im Bereich der Organisation und Finanzierung sozialer Projekte).

Eine Verbreitung von Crowdsourcing ist vor allem in der IT Industrie, insb. in der Internet-, Software- und IT-Service Branche zu beobachten. Ausschlaggebend hierfür ist ein stetig steigender Wettbewerbsdruck, der Softwareunternehmen kontinuierlich gezwungen hat, nach neuen Möglichkeiten zu suchen, um Produktions- und Entwicklungsprozesse kosteneffizienter $\mathrm{zu}$ gestalten. Infolgedessen wurde über die letzten Jahre hinweg kontinuierlich an der Industrialisierung, insbesondere der SoftwareEntwicklung gearbeitet, wobei Effizienzsteigerungen durch erhöhte Standardisierung, Automatisierung und Arbeitsteilung im Rahmen des Leistungserstellungsprozesses realisiert werden sollen (Bitkom, 2010). Hierbei geht es im Wesentlichen um die Standardisierung von Softwarekomponenten, sodass entsprechend die Erstellungsaktivitäten bzw. -prozesse auch standardisiert und (teilweise) automatisiert werden können. Um den individuellen Bedürfnissen der Kunden jedoch weiterhin gerecht zu werden, erfordert dies gleichzeitig eine modul- und komponentenorientierte Architektur von Software. Die Möglichkeiten zur verteilten Softwareentwicklung haben u.a. auch zur Auslagerung von Wertschöpfungsaktivitäten über das Internet geführt und entsprechende Arbeitsformen Digital Work und Crowd Work sind bereits seit über 10 Jahren beobachtbar.

Durch Crowdsourcing wird eine Arbeitsteilung im Rahmen der Softwareentwicklung ermöglicht, die bisherige Dimensionen überschreitet. Beim CrowdsourcingModell handelt es sich jedoch nicht lediglich um ein innovatives Konzept zur Verteilung und Durchführung von Unternehmensaufgaben, sondern vielmehr um eine gänzlich neue Art der Arbeitsform, mit welcher (teils radikale) Veränderungen sowohl auf der Unternehmens- als auch auf der Arbeitgeberseite einhergehen (Leimeister \& Zogaj, 2013). So ändern sich beispielsweise Kommunikations- und Koordinationsprozesse auf Seiten der Unternehmen, während sich aus Sicht der einzelnen Arbeitskraft im Wesentlichen die Art der Aufgabenbewältigung, die Arbeitsgestaltung und -bedingungen verändern. Vor diesem Hintergrund stellen sich nun mehrere Fragen: Wie genau „funktioniert“ Crowd Work? Welche Mechanismen stehen dahinter? Wie erfolgt der Leistungserstellungsprozess im Rahmen von Crowd Work bzw. Crowdsourcing? Was bedeutet Crowd Work für die beteiligten Crowd Worker - also für die Individuen, die hierbei Arbeitsleistung erbringen? Welche Folgen hat Crowd Work für die Beteiligten (Crowd Worker, Crowdsouring betreibende Unternehmen)?

Diese Fragen sollen in dem vorliegenden Beitrag basierend auf dem aktuellen Forschungs- und Wissensstand adressiert werden. Die Auseinandersetzung mit derartigen Fragestellungen scheint vor dem Hintergrund der voranschreitenden Digitalisierung der Arbeit in allen Bereichen unabdingbar. Die Softwarebranche nimmt hierbei zwar 
eine Vorreiterrolle ein - wie die Vergangenheit aber gezeigt hat, sind Veränderungen in diesem Sektor zumeist kennzeichnend für entsprechende bzw. darauf folgende Entwicklungen in anderen Sektoren gewesen. Bevor den aufgeführten Fragen nachgegangen wird, gilt es im ersten Schritt zunächst die Konzepte Crowdsourcing und Crowd Work genauer zu definieren.

\section{$2 \quad$ Was ist Crowdsourcing, was ist Crowd Work?}

Der Begriff „Crowdsourcing“ stellt eine Wortneuschöpfung aus den Wörtern „Crowd“ und „Outsourcing“ dar und geht auf Jeff Howe zurück, der diesen erstmals im Wired Magazine von 2006 verwendete (Howe, 2006). Durch diese Wortzusammensetzung wird ersichtlich, inwiefern sich der Begriff Crowdsourcing vom Outsourcing unterscheidet. Während unter dem Begriff Outsourcing eine Auslagerung einer definierten Aufgabe an ein Drittunternehmen oder eine bestimmte Institution bzw. einen Akteur verstanden wird, adressiert die Auslagerung im Falle des Crowdsourcings eben die „Crowd“, also eine undefinierte Masse an Menschen (Leimeister, 2012a). Crowdsourcing bezeichnet somit die Auslagerung von bestimmten Aufgaben eines Unternehmens oder im Allgemeinen einer Institution an eine undefinierte Masse an Menschen mittels eines offenen Aufrufs, welcher zumeist über das Internet erfolgt. In einem Crowdsourcing-Modell gibt es immer die Rolle des Auftraggebers - der als Crowdsourcer bezeichnet wird - sowie die Rolle der undefinierten Auftragnehmer, also die Crowd oder in Analogie zum erstgenannten Begriff die Crowdsourcees bzw. Crowd Worker. Die Durchführung von Crowdsourcing Initiativen erfolgt indessen über eine Crowdsourcing-Plattform, die intern aufgesetzt werden kann oder von einem Crowdsourcing Intermediär bereitgestellt wird. Fig. 1 stellt die unterschiedlichen Rollen zusammenfassend dar. 

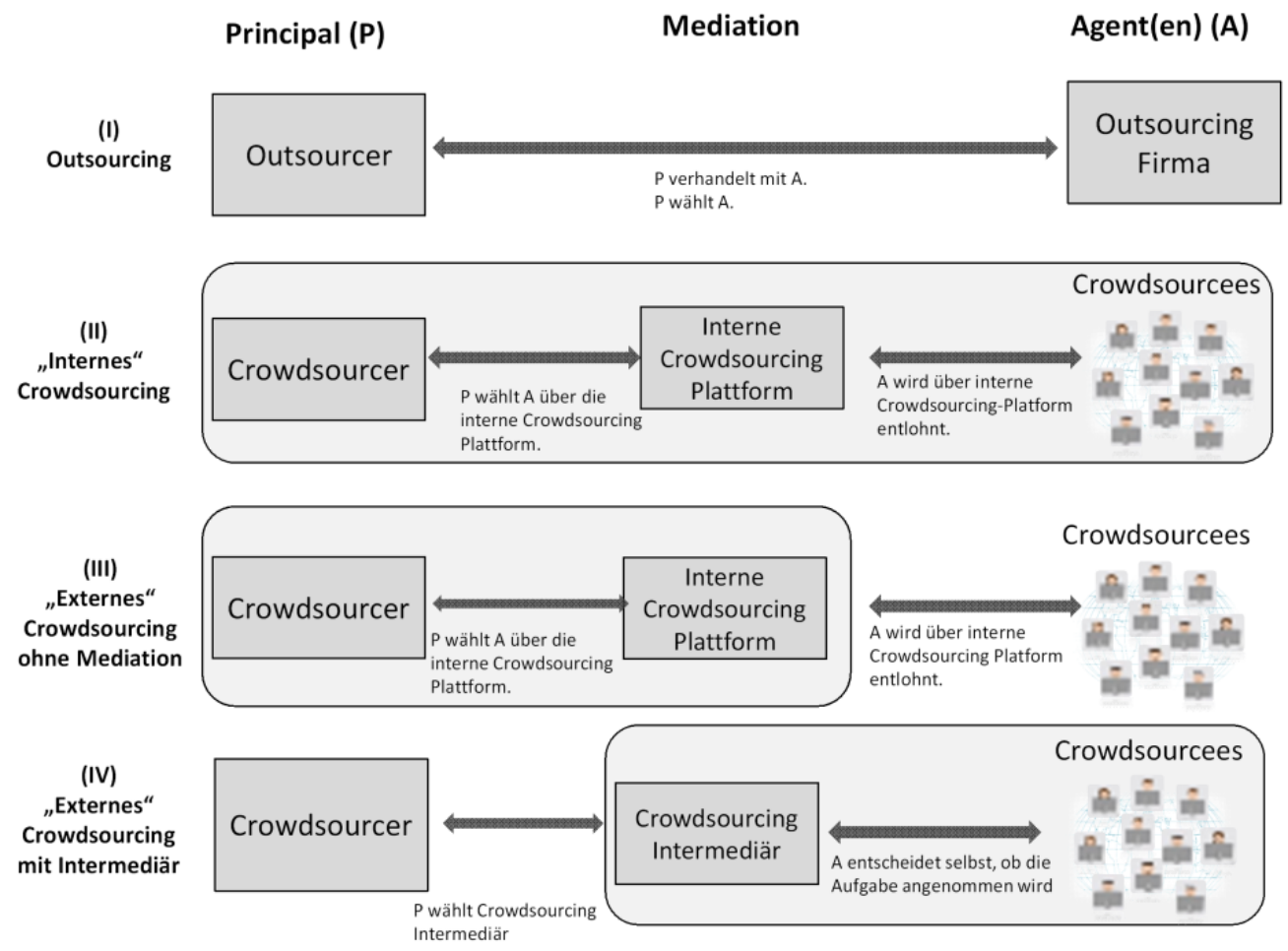

Fig. 1. Rollen und Mediation im Crowdsourcing-Modell. Quelle: In Anlehnung an (Hoßfeld, Hirth, \& Tran-Gia, 2012, S. 206).

Es ist zunächst zwischen ,internem“ Crowdsourcing und „externem“ Crowdsourcing zu unterscheiden. Im ersten Fall fungiert die unternehmensinterne Belegschaft bzw. Teile hiervon als Crowd. Demzufolge ist jede(r) Mitarbeiter(in) des betreffenden Bereichs des Unternehmens als Crowd Worker zu bezeichnen. Hierbei dient eine unternehmensintern aufgesetzte Plattform (Intranet/Internet-basierte Plattform) als Crowdsourcing-Plattform, über welche die Crowd (= interne Belegschaft) Beiträge erbringen kann. Beim externen Crowdsourcing hingegen besteht die Crowd aus beliebigen Individuen, die nicht in einem Zusammenhang mit dem Unternehmen bzw. Crowdsourcer stehen müssen. Dies sind zumeist unternehmensexterne Personen theoretisch kann hierbei also jede Person weltweit mit einem Internetanschluss als Crowdsourcee bzw. Crowd Worker fungieren. Die Crowdsourcing-Plattform kann hierbei einerseits vom Unternehmen selbst aufgesetzt, verwaltet und gemanagt werden. Andererseits besteht die Möglichkeit, Crowdsourcing Intermediäre heranzuziehen, die selbst eine (aktive) Crowd - bestehend aus einer Community von Crowd Workern - aufbauen und Crowdsourcing betreibenden Unternehmen die Möglichkeit bieten, ihre Aufgaben über die aufgesetzte Crowdsourcing-Plattform auszulagern. Die zwei aufgeführten Vorgehensweisen (externes und internes Crowdsourcing) schließen sich nicht gegenseitig aus, sondern es sind fließende Übergänge denkbar und auch in der Praxis bereits beobachtbar.

Crowdsourcing beschränkt sich somit keinesfalls auf die Verlagerung von Aufgaben in die Unternehmensumwelt, es kann auch unternehmensintern Aufbau- und Ablauforganisation verändern. Diese neue Art der Arbeitsorganisation bezeichnen wir 
als Crowd Work. Crowd Work erscheint als Wertschöpfungs- und Koordinationsmodell zwischen Markt und Hierarchie (vgl. Fig. 2). So hat bspw. IBM mit seinem „Liquid“ Programm eine Initiative aufgesetzt, die langfristig die Verlagerung von 8.000 Arbeitsplätzen in eine unternehmensinterne Crowd ermöglichen soll und bei der die Aufgaben über die Liquid-Plattformen ein effektives internes Crowdsourcing ermöglichen soll, um Mitarbeitern mit freien Kapazitäten bessere Arbeitsmöglichkeiten zu geben.

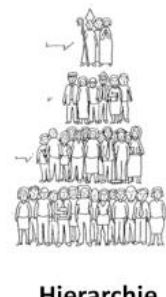

Hierarchie

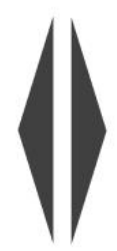

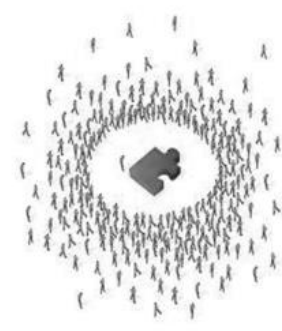

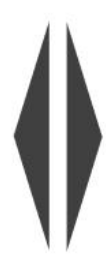

Crowd Work

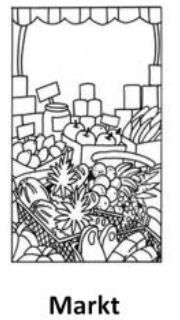

Markt

Fig. 2. Crowd Work als Wertschöpfungs- und Koordinationsmodell zwischen Markt und Hierarchie. Quelle: Eigene Darstellung.

\section{$3 \quad$ Wo kommt Crowd Work bereits zum Einsatz?}

Moderne Informations- und Kommunikationssysteme, allen voran das Internet, schaffen die technischen Voraussetzungen für eine unternehmensübergreifende $\mathrm{Zu}$ sammenarbeit mit vielen externen Beitragenden bzw. der Crowd (Martin, Lessmann, \& Voß, 2008). In diesem Kontext wird Crowd Work als eine neue und auch bereits ernstzunehmende Arbeitsorganisationsform betrachtet. Diese ermöglicht es, auf eine Vielzahl von Arbeitskräften (Crowd Worker) - die durch unterschiedliche Wissensstände, Fähigkeiten, Erfahrungen und Hintergründe gekennzeichnet sind - zur Erstellung der Leistungen zurückzugreifen. Crowd Work kommt mittlerweile im Rahmen unterschiedlichster Aktivitäten innerhalb von Leistungserstellungsprozessen zum Tragen (vgl. Fig. 3).

So nutzen Softwareunternehmen Crowdsourcing-Plattformen wie beispielsweise TopCoder (topcoder.com) oder CrowdCode (crowdco.de) für die Programmierung von Softwareapplikationen durch Crowd Worker. Über Plattformen wie testCloud (testcloud.de), uTest (utest.com), testHub (testhub.com) oder PassBrains (pass- 
brains.com) können Softwareunternehmen auch das Testen ihrer Software an eine Crowd auslagern (Zogaj, Bretschneider, \& Leimeister, 2013). Für die vorgeschaltete Analyse und Definition von Anforderungen können Crowd-Befragungen durchgeführt werden, während Crowd-Ideation Plattformen für die Gestaltung und den Entwurf einer Softwareapplikation herangezogen werden können. Querschnitts- und Unterstützungsaufgaben - wie z. B. Eingabe, Strukturierung und Bereinigung von Datensätzen - werden hingegen über Plattformen wie Amazon Mechanical Turk (mturk.com) und Elance (elance.com) an die Crowd ausgelagert.

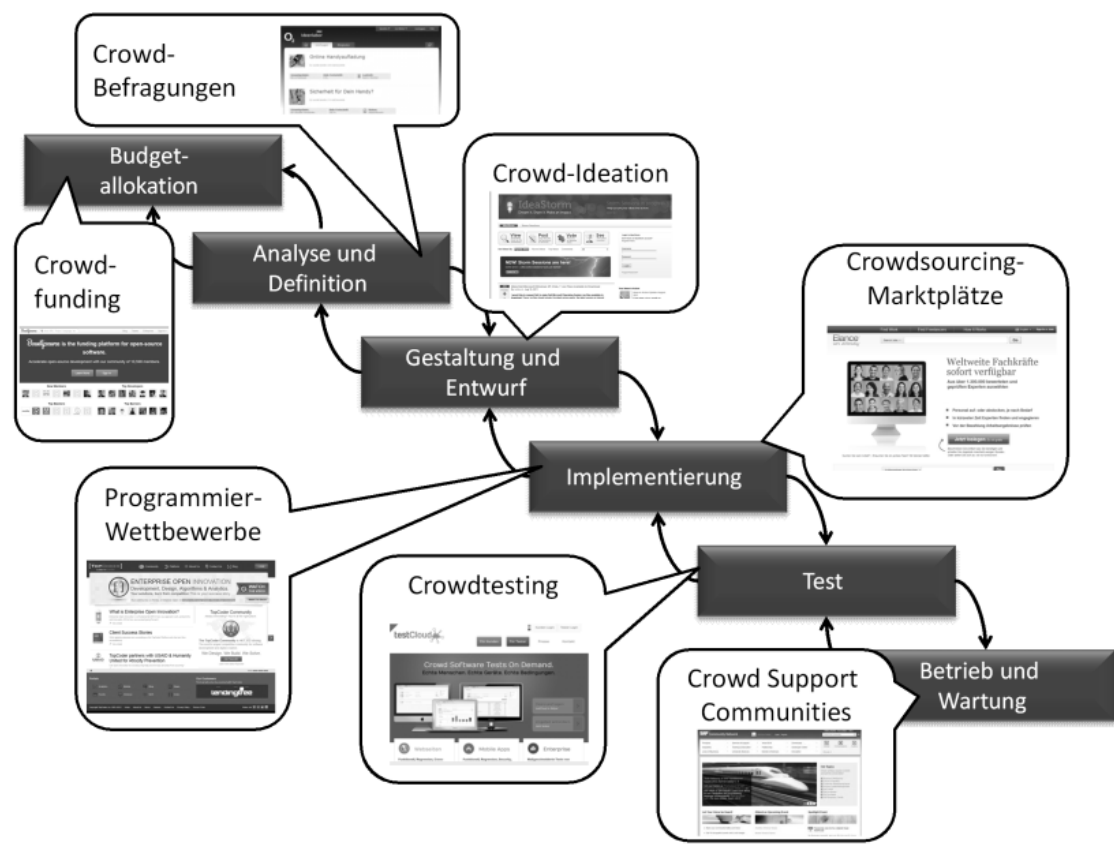

Fig. 3. Crowd Work in der IT- und Softwareentwicklung. Quelle: Eigene Darstellung.

\section{$4 \quad$ Wie ,funktioniert" Crowd Work?}

Mit der Auslagerung von unternehmensinternen Aktivitäten an die Crowd gehen unterschiedliche Herausforderungen in Bezug auf das Management der (Zusammen-) Arbeitsprozesse einher, da sich hierdurch zunehmend die Grenzen einer Unternehmung auflösen und sich Unternehmen zur Leistungserstellung nicht nur der internen Belegschaft, sondern für nahezu jede Aktivität auch der externen Crowd bedienen können. Insofern stellt sich zunächst die Frage, was die Kernleistungen eines Unternehmens sind und welche Aktivitäten qualitativ hochwertiger, schneller oder kostengünstiger von der Crowd durchgeführt werden können. In diesem Zusammenhang ist somit zu klären, „wie“ Aufgaben an die Crowd ausgelagert werden können bzw. allgemeiner gefragt, „wie“ implementiert werden kann. Damit ergeben sich zunächst Herausforderungen hinsichtlich des Managements des Crowdsourcing-Prozesses sowie der Steuerung und Kontrolle von Arbeitsaktivitäten (Geiger, Seedorf, Schulze, Nickerson, \& Schader, 2011; Jain, 2010). Von besonderer Bedeutung sind hierbei auch die Arbeitsbedingungen und die Arbeitsgestaltung im Rahmen von Crowd Work 
- das heißt die Arbeitsformen in der Crowd, die Anreizstrukturen, Arbeitsbedingungen und entsprechend Entlohnungsmodelle für Crowd Worker.

\subsection{Management des Crowdsourcing-Prozesses}

Crowdsourcing betreibende Unternehmen stehen zunächst vor der Herausforderung $\mathrm{zu}$ entscheiden, welche internen Leistungserstellungsaktivitäten an die Crowd ausgelagert werden (können). Die Literatur und Praxis verdeutlichen, dass nahezu jegliche Wertschöpfungsaktivitäten vom Crowdsourcing betroffen sein können. Damit interne Arbeitspakete von Crowd Workern erfolgreich erledigt werden können, müssen diese konkretisiert, detailliert beschrieben und zumeist in kleine (Arbeits-) Einheiten zerlegt werden (Arbeits- bzw. Aufgabendekomposition). Das für die Durchführung der Teilaufgaben benötigte Know-how ist entsprechend gering, sodass dadurch viele, auch nicht für eine bestimmte (größere) Aufgabe hochqualifizierte Individuen an der Aufgabenbearbeitung mitwirken können. Diese Vorgehensweise ist mit den Prinzipien des Taylorismus vergleichbar. Ein Ziel hierbei ist, die Arbeitsproduktivität durch Standardisierung und Zerlegung komplexer Arbeitsvorgänge in kleinere Einzeltätigkeiten (und damit auch durch stärkere Arbeitsteilung) zu steigern. Diese kleineren und häufig auftretenden Einzeltätigkeiten können dann von (ggf. auch weniger qualifizierten bzw. leichter und schneller anlernbaren) Arbeitskräften unter Ausnutzung von Lern-, Größen- und Verbundeffekten effektiver bzw. effizient bearbeitet werden und die Produktivität und Geschwindigkeit der Gesamtleistungserstellung so ggf. gesteigert werden. Analog zur Industrialisierung von Produktionsprozessen entfällt ein Großteil des Aufwandes bei der Aufgabenbearbeitung auf die Arbeitsplanung, -steuerung und -koordination. Crowdsourcing- bzw. Prozessmanager, die die disponierende Einteilung übernehmen und die Arbeitsprozesse im Rahmen von Crowdsourcing steuern und kontrollieren, haben andere Aufgaben zu bewerkstelligen als ,konventionelle“ Projekt- bzw. Prozessmanager.

Eine zentrale Herausforderung im Rahmen von Crowd Work ist es, den Crowdsourcing-Prozess mit allen dazugehörigen Aktivitäten zu planen, zu implementieren, zu steuern und zu kontrollieren. Hierzu ist es dementsprechend auch notwendig, alle mit den einzelnen Phasen verbundenen - Aktivitäten bzw. Handlungsoptionen genau zu erörtern. Der Crowdsourcing-Prozess kann idealtypisch in fünf Phasen unterteilt werden (vgl. Fig. 4): Während in der ersten Phase im Wesentlichen die Zerlegung der Arbeitspakte in Teilaufgaben sowie die Bestimmung der Lösungs- bzw. Aufgabenanforderungen erfolgt, wird in der zweiten Phase bestimmt, welche Crowd-worker (alle oder nur eine Teilmenge der Crowd) dann im dritten Schritt wie die jeweiligen Aufgaben bewältigen sollen. In der vierten Phase werden die eingereichten Lösungen bzw. Beiträge (zu einer Gesamtlösung) bewertet und zusammengetragen, sodass darauf basierend schließlich die Vergütung der Crowd Worker erfolgen kann. 


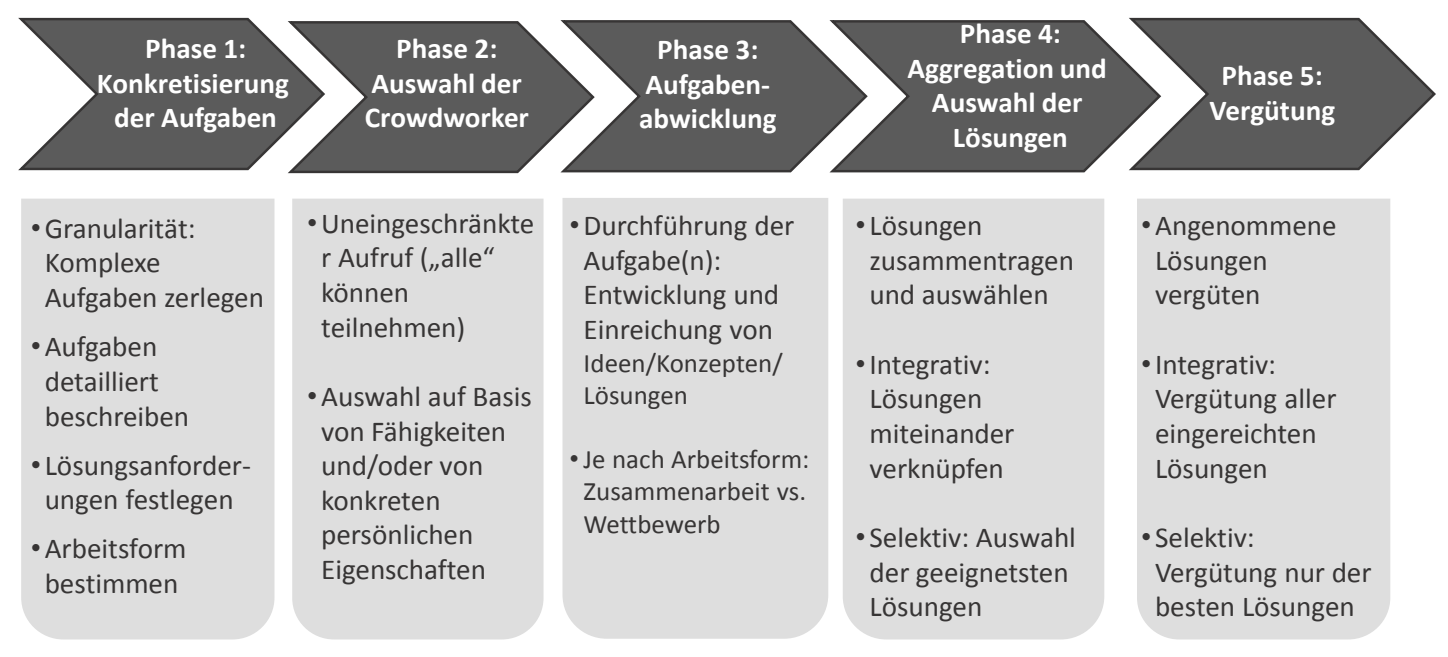

Fig. 4. Phasen und Maßnahmen im Crowdsourcing-Prozess. Quelle: Eigene Darstellung.

\subsection{Steuerung und Kontrolle von Crowdaktivitäten}

Die gezielte Steuerung und Kontrolle von Crowdaktivitäten („Crowd Governance") wird als eine der wesentlichen Herausforderungen im Rahmen von Crowdsourcing betrachtet. Dieses wird dadurch begründet, dass eine „unkontrollierte“ Crowd nicht vereinbarte Ziele erreichen kann. Beispielsweise kann es dazu kommen, dass Crowd Worker bestimmte Aufgaben nicht innerhalb einer vorgegebenen Zeit durchführen und der Crowdsourcing-Intermediär seinem Kunden (Crowdsourcer) die Lösungen nicht bereitstellen kann. Die Steuerung und Kontrolle im Rahmen von Crowdsourcing umfasst alle Maßnahmen und Vorgehensweisen zum effektiven Management der Crowd. „Kleinere“ Crowdsourcing Projekte (z. B. einfaches Brainstorming durch die Crowd) erfordern eventuell weniger Kontrollaufwand, wohingegen im Rahmen komplexerer Crowdsourcing Projekte umfassende Kontroll- und Steuerungsmechanismen zu implementieren sind. In der Forschung mangelt es aber an Untersuchungen explizit zu Steuerungs- und Kontrollmechanismen. Table 1 fasst die wesentlichen Steuerungs- und Kontrollmechanismen im Rahmen von Crowdsourcing zusammen. 
Aufgabengestaltung

Gestaltung geeigneter Strukturen, die Aufgabenbearbeitungsprozesse effektiv unterstützen. Hierbei geht es insbesondere um die Konkretisierung der Aufgaben bzw. um das Herunterbrechen der Aufgaben in Teilaufgaben und die entsprechende Aggregation der Teillösungen zu einer Gesamtlösung.

Feedbackmechanismus

Etablierung von Maßnahmen, mithilfe derer die Crowd Worker Feedback vom Crowdsourcer oder vom Crowdsourcing-Intermediär erhalten können. Das Feedback kann sich sowohl auf die eigentliche Arbeit bzw. Arbeitsleistung, auf einzelne Aufgaben oder auf allgemeine Sachverhalte im Rahmen der Crowdsourcing Plattform beziehen.

Anreizstrukturen

Etablierung von Strukturen und Maßnahmen, die die Motive der Crowd Worker adressieren und diese entsprechend dazu motivieren, auf der Crowdsourcing Plattform aktiv zu sein. Die Anreizstrukturen orientieren sich an den Bedürfnissen und somit sowohl an extrinsischen als auch an intrinsischen Motiven.

Management der Lösungen \& Qualitätssicherung

Etablierung von Mechanismen, mithilfe derer die Qualität eingereichter Lösungen bewertet werden kann. Die Bewertung der Lösungen über zuvor definierte Lösungsanforderungen ist ein gängiger Ansatz, der jedoch von Crowdsourcing Initiative zu Crowdsourcing Initiative variiert. Neben diesem Vorgehen werden in der Literatur noch drei weitere Ansätze vorgestellt: (1) Bewertung der eingereichten Lösungen durch andere Crowd Worker (z.B. anhand von 5-Star-Ratings); (2) Vermischung von tatsächlichen Aufgaben und Testaufgaben, um dadurch zu kontrollieren, ob die Crowd Worker die Aufgaben auch tatsächlich lösen; (3) Iterative Ansätze, im Rahmen derer mehrere Crowd Worker dieselbe Aufgabe erledigen. Sofern zwei (oder mehr) Crowdworker zur selben oder ähnlichen Lösung kommen, kann angenommen werden, dass die betreffende Lösung geeignet ist.

Mitgliedermanagement

Etablierung von Mechanismen, mithilfe derer die Qualität der Arbeit und der Crowd Worker innerhalb von Crowdsourcing Plattformen sichergestellt werden kann. Hierzu zählen Einarbeitungsmaßnahmen und das Bereitstellen von Diskussionsforen in der Community. Hinzu kommen auch Maßnahmen und Strukturen, mithilfe derer Crowd Worker Kontakt zu dem Plattformbetreiber bzw. dem Crowdsourcer aufnehmen können.

Table 1. Steuerungs- und Kontrollmechanismen im Bereich Crowdsourcing. Quelle: Eigene Darstellung in Anlehnung an (Jain, 2010, S. 3ff.; Pedersen et al., 2013).

\subsection{Arbeiten in der Crowd: Arbeitsformen, Motive und Anreizstrukturen}

Beim Crowdsourcing initiiert der Crowdsourcer den Crowdsourcing-Prozess, definiert und konkretisiert die zu bearbeitenden Aufgaben, bestimmt die Anreizstrukturen und verwertet die Lösungen, während die Crowd Worker die bereitgestellten Aufgaben auswählen und bearbeiten. Die Bearbeitung der Aufgaben durch Crowd Worker erfolgt in der dritten Phase eines idealtypischen Crowdsourcing-Prozesses. Hier stellt sich jedoch die Frage, wie genau die Arbeit auf Crowdsourcing-Plattformen erfolgt, da sich Arbeitsprozesse in Online-Umgebungen mit einer großen Zahl an Akteuren von klassischen unternehmensinternen Arbeitsprozessen strukturell unterscheiden können.

Grundsätzlich kann in Bezug auf Arbeitsformen zwischen zwei Ansätzen unterschieden werden: wettbewerbsbasierter Ansatz und zusammenarbeitsbasierter Ansatz. Im ersteren Fall stehen die Crowd Worker entweder in einem zeitlichen (der Crowd Worker, der die Aufgabe als Erster erfolgreich erfüllt, wird ent- bzw. belohnt) oder ergebnisorientierten (nur der oder die Crowd Worker mit der besten Lösung wird/werden be- bzw. entlohnt) Wettbewerb zueinander. In diesem Kontext arbeiten 
die Crowd Worker unabhängig voneinander und generieren entsprechend Lösungen individuell. Beim zusammenarbeitsbasierten Ansatz erarbeiten hingegen mehrere Crowd Worker gemeinsam eine Lösung zu einer bestimmten Aufgabe. Hierbei reicht ein Crowd Worker einen Beitrag ein und andere Crowd Worker, die mitwirken wollen, haben auf der Plattform die Möglichkeit, den eingereichten Beitrag zu verändern und auch zu erweitern. Dieses geschieht zumeist anhand einer Kommentarfunktion, über die die Crowd Worker miteinander über die Lösung diskutieren können. Der Output ist dann eine gemeinsam erarbeitete Lösung (vgl. Fig. 5).

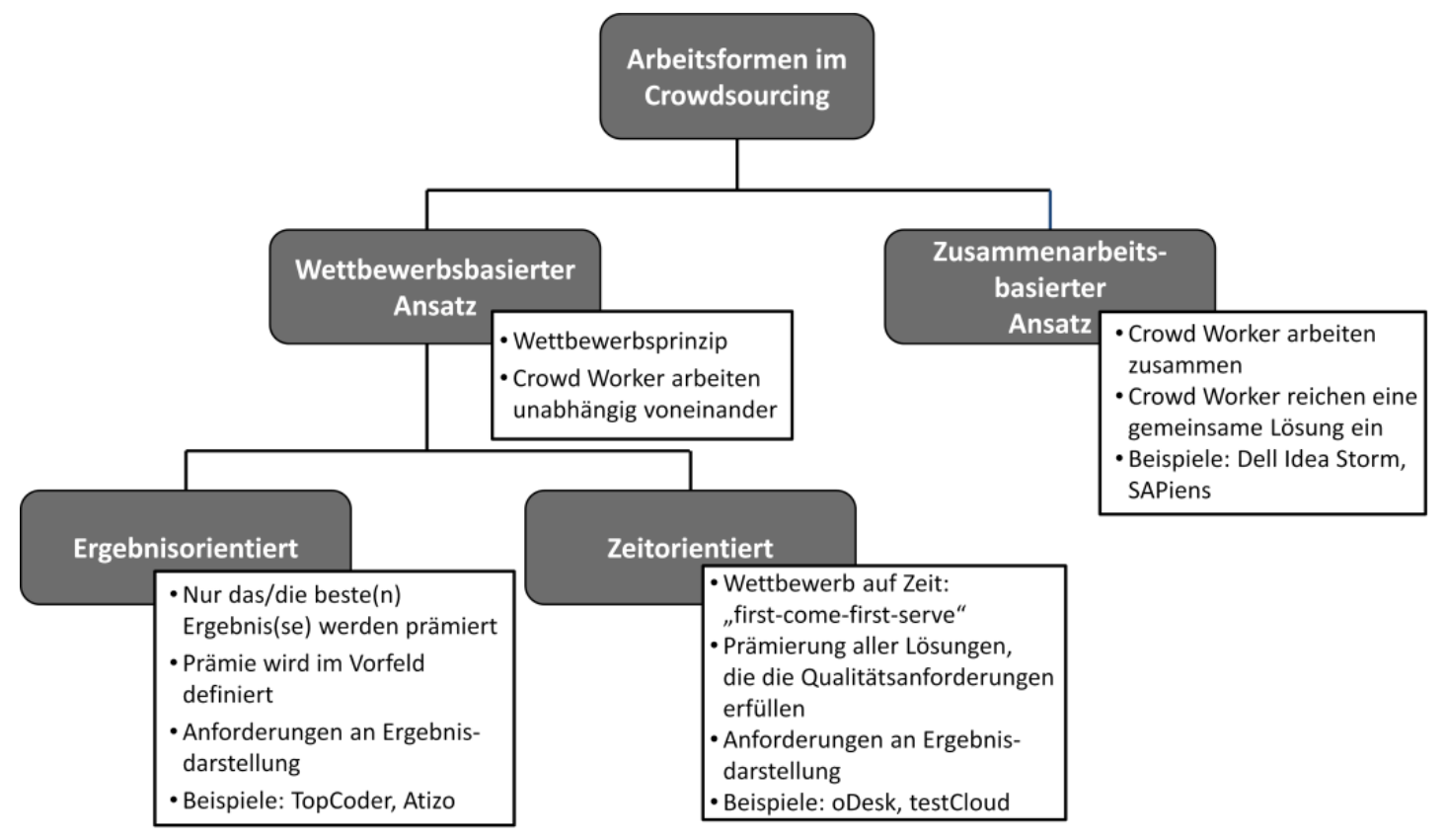

Fig. 5. Arbeitsformen im Crowdsourcing. Quelle: Eigene Darstellung.

Im Vergleich zu „klassischen“ Arbeitsvorgängen, im Rahmen derer Arbeitsaufgaben zumeist vorgegeben bzw. von Vorgesetzten delegiert oder zugeordnet werden, bestimmen beim Crowdsourcing die Crowd Worker selbst, welche und auch wie viele der auf einer Crowdsourcing-Plattform ausgeschriebenen Aufgaben sie entgegennehmen bzw. erledigen. Aber was genau treibt Crowd Worker dazu, an Crowdsourcing Initiativen teilzunehmen?

Unterschiedliche Studien zeigen auf, dass intrinsische Motive wie der soziale Austausch, die Möglichkeit zur Erweiterung der individuellen Fähigkeiten und die Freude an der (Crowd-) Arbeit sowie (je nach Crowdsourcing-Modell) die Möglichkeit auf höheren Grad an Selbstbestimmung bei der Arbeit (durch eigene Wahl der Crowd Work Aktivitäten, des Zeitpunkts, Ortes etc.) eine wesentliche Rolle spielen. Prämienbasierte und monetäre Entlohnungen (extrinsische Motive) stellen jedoch den primären Anreizfaktor für Crowd Worker dar. Darüber hinaus wird eine hohe Eigenbestimmung bei der Wahl und Art der Tätigkeit als Crowd Worker als positiv dargestellt. Entsprechend sind in der Praxis unterschiedliche Entlohnungs- bzw. Vergütungsmodelle vorzufinden. Die Prämien bzw. Entgelte variieren stark, abhängig von der Arbeitsform und der Art der Aufgaben. Während Crowd Worker für manche Auf- 
gaben mit wenigen Eurocents entlohnt werden, gibt es auch mehrere Crowdsourcing Initiativen, in deren Rahmen Preisgelder von bis zu 100 Tsd. Euro oder Dollar vergeben werden. In der folgenden Tabelle werden einige Entlohnungsformen und ihre Besonderheiten vorgestellt.

\begin{tabular}{|l|l|l|}
\hline Plattform & System & Anreizsystem / Entlohnung \\
\hline $\begin{array}{l}\text { Amazon } \\
\text { Mechanical } \\
\text { Turk }\end{array}$ & $\begin{array}{l}\text { Crowdsourcer } \\
\text { gibt Preis vor }\end{array}$ & $\begin{array}{l}\text { Fixe Zahlung pro Aufgabe. Bezahlung erfolgt nur, wenn Lösung vom } \\
\text { Crowdsourcer akzeptiert wird. Der durchschnittliche Stundenlohn liegt bei circa } \\
1,25 \text { \$. Es gibt nur wenige Aufgaben, die spezielle/s Fähigkeiten/Wissen } \\
\text { voraussetzen. }\end{array}$ \\
\hline Spreadshirt & $\begin{array}{l}\text { Crowdworker } \\
\text { gibt Preis vor }\end{array}$ & $\begin{array}{l}\text { Crowdworker bietet Unternehmen ein T-Shirt-Design zu einem selbst } \\
\text { festgelegten Preis zum Kauf an und erhält bei jedem verkauften T-Shirt eine } \\
\text { Gewinnbeteiligung. }\end{array}$ \\
\hline InnoCentive & Wettbewerb & $\begin{array}{l}\text { InnoCentive organisiert Wettbewerbe für Unternehmen, in denen nach } \\
\text { Lösungen in einem speziellen Bereich gesucht wird, z. B. Produktentwicklung } \\
\text { oder Wissenschaft. Die Entlohnung erfolgt über Auszeichnungen oder } \\
\text { finanziellen Ausgleich. Die Bezahlung hängt dabei vom Schwierigkeitsgrad ab } \\
\text { und kann bis zu 100.000 \$ betragen. }\end{array}$ \\
\hline IBM \\
Liquid & Punktesystem & $\begin{array}{l}\text { Crowdworker erhalten für ihre Teilnahme an der Ausschreibung so genannte } \\
\text { „Liquid Points“. Diese belegen ihre Teilnahme an einem bestimmten } \\
\text { Wettbewerb und dienen der Verbesserung der Community-internen } \\
\text { Reputation. Auf Basis der Punte wird eine unternehmensinterne Rangliste } \\
\text { geführt. Ein höherer Rang kann die Chancen auf eine Auswahl bei anderen } \\
\text { Ausschreibungen verbessern. }\end{array}$ \\
\hline
\end{tabular}

Table 2. Beispiele zur Entlohnung von Crowd Workern. Quelle: Eigene Darstellung.

Insbesondere fundierte und empirisch gesicherte Erkenntnisse über zugrunde liegende Motive und das Erleben derartiger Arbeit von Crowd Workern können dazu eingesetzt werden, bessere Crowd Work Prozesse, Plattformen und Arbeitsbedingungen zu entwickeln (Leimeister et al. 2009) und somit zu „besserer digitaler Arbeit““ der Zukunft genutzt werden.

\section{$5 \quad$ Welche Chancen und Risiken birgt Crowd Work?}

Crowdsourcing ist über die letzten Jahre hinweg für viele Unternehmen eine ernstzunehmende Alternative zur Aufgabenbearbeitung geworden. Nicht nur Softwareunternehmen, sondern auch Unternehmen aus anderen Bereichen (bspw. IBM, BMW, Audi, MC Donald's, Otto, Henkel, Tchibo, Sennheiser etc.) neigen dazu, unterschiedlichste Aufgaben an Crowds auszulagern - von Innovations- (bspw. Ideengenerierung) über zu Marketing- (bspw. Erstellen von Logos, Werbeslogans) bis hin zu allgemeinen Unterstützungsaufgaben (bspw. Durchführung von Kalkulationen) (Leimeister, 2012b; Leimeister \& Zogaj, 2013). Zahlreiche Forscher messen der Öffnung interner Unternehmensprozesse für die Crowd ein hohes Potenzial für Unternehmen bei. Manche beziehen sich in diesem Zusammenhang auf das enorme Wissenspotenzial der Crowd (Howe, 2008), während andere von der „Erreichung einer neuen Evolutionsstufe hinsichtlich der (unternehmerischen) Wertschöpfung“ (Hammon \& Hippner, 2012) durch die Nutzung des Potenzials der Crowd sprechen. 
Auf der anderen Seite ergeben sich auch für Crowd Worker unterschiedliche Möglichkeiten und Chancen, die im Rahmen „klassischer“ Arbeitsformen nur eingeschränkt realisierbar sind.

Im Gegensatz dazu werden in vielen Berichten auch insbesondere die Risiken, die mit Crowd Work sowohl für Crowd Worker (internes Crowdsourcing) als auch für Unternehmen einhergehen kritisch diskutiert. So wird in einzelnen Beiträgen vor der Entstehung von „digitalen Ausbeuterbetrieben“ (engl. „digital sweatshops“) gewarnt, da die Entlohnung für die Crowd Worker zum Teil sehr gering ausfallen kann und zudem nicht sicher ist (siehe bspw. Zittrain, 2009). Für Unternehmen besteht vor allem die Gefahr, dass unternehmensinternes Wissen durch Crowdsourcing nach außen fließt oder dass Schwierigkeiten in Bezug auf die Kontrolle der Arbeitsprozesse entstehen. Nachfolgend sind die wesentlichen Chancen und auch Risiken, die sowohl für Crowdsourcing betreibende Unternehmen als auch für Crowd Worker mit Crowd Work einhergehen, tabellarisch aufgeführt (Fig. 6).

\begin{tabular}{|l|l|}
\hline Chancen & Chancen \\
\hline - Zugriff auf einen (immensen) Wissens- und Kompetenzpool & - Entlastung interner Mitarbeiter durch Konzentration auf \\
wesentliche Aufgaben
\end{tabular}

Fig. 6. Chancen und Risiken für Crowdsourcer und Crowd Worker. Quelle: Eigene Darstellung.

Trotz der langen Bilanz von Vor- und Nachteilen von Crowdsourcing auf unterschiedlichen Ebenen bleibt festzuhalten, dass es an belastbaren wissenschaftlichen Erkenntnissen in großen Teilen fehlt. Welche Risiken und Potenziale sich mit der Implementierung von Crowdsourcing tatsächlich ergeben, welche Wirkprinzipien hier wann wie zu Grunde liegen, welche Auswirkungen auf Menschen, Organisationen und Märkte sich hieraus unter welchen Rahmenbedingungen ergeben, kann mit den aktuellen Erkenntnissen zu diesem Themenbereich noch nicht fundiert bestimmt oder vorhergesagt werden. 


\section{Crowdsourcing und Crowd Work - Bedeutung für die Wirtschaftsinformatik}

Unser Leben wird immer stärker von digitalen Technologien beeinflusst. Die Digitalisierung verändert sowohl unsere Gesellschaft als auch uns als Individuen - und damit unter anderem auch die Art wie wir arbeiten bzw. wie Unternehmen ihre Arbeits- und Leistungserstellungsprozesse organisieren. Mit Crowdsourcing und Crowd Work ist ein innovatives Modell zur Organisation von Leistungserstellungsprozessen entstanden. Es ist davon auszugehen, dass immer mehr Unternehmen das Konzept heranziehen werden, um auf ein großes Reservoir an Arbeitskräften schnell und gezielt zugreifen zu können. Eine wachsende Verbreitung von Crowd Work impliziert nicht nur vereinzelte, kurzfristige Änderungen einzelner Unternehmen oder einzelner Crowds - vielmehr kann aus der Verbreitung dieses Arbeitsorganisationskonzeptes mittel- bis langfristig ein disruptiver Wandel von Organisations- und Arbeitsstrukturen resultieren. Dementsprechend gilt es, dieses Konzept und die damit verbundenen gesellschaftlichen und wirtschaftlichen Veränderungen auf Makro- (gesamtwirtschaftlich betrachtet), Meso- (bezogen auf eine einzelne Organisation und deren Prozesse) und Mikro-Ebene (Beschäftigungs- und Arbeitsverhältnisse auf Individualebene) eingehend zu untersuchen.

Im vorliegenden Beitrag konnte ein Ausschnitt davon gezeigt werden, wie auf der einen Seite die Prozesse im Rahmen von Crowd Work gestaltet, kontrolliert und gesteuert werden können. Auf der anderen Seite wurde auch die Perspektive der Crowd Worker eingenommen. In diesem Zusammenhang erfolgte eine Auseinandersetzung mit den Arbeitsbedingungen und -formen im Rahmen von Crowd Work. Bezugnehmend auf alle im Beitrag adressierten Aspekte handelt es sich derzeit noch um ,erstes, frühes Wissen", da die Forschung im Bereich Crowd Work bzw. Crowdsourcing noch in den Anfängen ist. Es ist bei weitem noch nicht klar, warum wann wie welche Arbeitsprozesse, Steuerungs- und Kontrollmechanismen oder Anreizstrukturen in unterschiedlichen Kontexten und Branchen auszugestalten bzw. zu implementieren sind. Auch sind die genauen Arbeitsbedingungen und Arbeitsverhältnisse von Crowd Workern weitestgehend unerforscht. So sind beispielsweise die Arbeitszeiten zumeist nicht konkret geregelt oder fixiert, genauso wenig wie die Beschäftigungsdauer (befristet oder unbefristet). Nicht spezifiziert sind weiterhin Mitbestimmungsrechte, Qualifizierungsansprüche und ähnliche Sachverhalten, die im Rahmen eines klassischen Arbeitsverhältnisses zumeist strikt geregelt sind. Hier stellt sich insbesondere die Frage, wie ,faire Arbeitsbedingungen für Crowd Worker" geschaffen und umgesetzt werden können.

Die möglichen gesellschaftlichen Auswirkungen von Crowdsourcing, auch über die IT-Branche hinaus, können sehr weitreichend sein. Die Chancen und das Potenzial von Crowdsourcing für Organisationen und Menschen zu heben - ohne die Nachteile und Gefahren wahr werden zu lassen -; die dahinter liegenden Wirkprinzipien zu verstehen, um daraus Lösungsinformationen und Konzepte für eine bessere Zukunft von Organisationen und Menschen zu erarbeiten und Wertschöpfung und Wohlstand zu sichern, sind unseres Erachtens Aufgaben der Wirtschaftsinformatik. Teil dieses Wandels zu sein und diesen in Richtung wettbewerbsfähiger, nachhaltiger und vor 
allem ,guter“ Arbeit und Wertschöpfung in und mit Crowds mitzugestalten und zu lenken, ist eine gesellschaftlich bedeutsame Chance für die Wirtschaftsinformatik der Zukunft.

\section{Referenzen}

1. Bitkom. (2010). Industrielle Softwareentwicklung - Leitfaden und Orientierungshilfe.

2. Bundesministerium für Bildung und Forschung. (2013). Das Wissenschaftsjahr 2014 - Die digitale Gesellschaft.

3. Geiger, D., Seedorf, S., Schulze, T., Nickerson, R., \& Schader, M. (2011). Managing the Crowd: Towards a Taxonomy of Crowdsourcing Processes. Paper presented at the Proceedings of the Seventeenth Americas Conference on Information Systems, Detroit, Michigan.

4. Hammon, L., \& Hippner, H. (2012). Crowdsourcing. Wirtschaftsinformatik, 54(3), 165-168.

5. Hoßfeld, T., Hirth, M., \& Tran-Gia, P. (2012). Aktuelles Schlagwort: Crowdsourcing. Informatik Spektrum, 35(3), 204-208.

6. Howe, J. (2006). The Rise of Crowdsourcing. Wired Magazine, 14(6), 1-4.

7. Howe, J. (2008). Crowdsourcing: Why the Power of the Crowd is Driving the Future of Business. New York.

8. Jain, R. (2010). Investigation of Governance Mechanisms for Crowdsourcing Initiatives, AMCIS 2010 Proceedings.

9. Leimeister, J. M., Huber, M., Bretschneider, U.; Krcmar, H. (2009): Leveraging crowdsourcing: activation-supporting components for IT-based ideas competition. Journal of Management Information Systems (JMIS), 26 (1), 197-224.

10. Leimeister, J. M. (2012a). Crowdsourcing. Zeitschrift für Controlling und Management (ZFCM), 56(6), 388-392.

11. Leimeister, J. M. (2012b). Dienstleistungsengineering und -management. Berlin, Heidelberg: Springer Gabler.

12. Leimeister, J. M., \& Zogaj, S. (2013). Neue Arbeitsorganisation durch Crowdsourcing: Eine Literaturstudie. Düsseldorf: Hans Böckler Stiftung.

13. Martin, N., Lessmann, S., \& Voß, S. (2008). Crowdsourcing: Systematisierung praktischer Ausprägungen und verwandter Konzepte, Tagungsband der Multi-Konferenz Wirtschaftsinformatik (MKWI'08). München.

14. Pedersen, J., Kocsis, D., Tripathi, A., Tarrel, A., Weerakoon, A., Tahmasbi, N., et al. (2013). Conceptual Foundations of Crowdsourcing: A Review of IS Research, 2013 46th Hawaii International Conference on System Sciences (pp. 579-588).

15. Unterberg, U. (2010). Crowdsourcing. In D. Michelis \& T. Schildhauer (Eds.), Social Media Handbuch: Theorien, Methoden, Modelle (pp. 121-135). Baden-Baden.

16. Zittrain, J. (2009). Work the New Digital Sweatshops. Retrieved 13.10.2013, from http://www.thedailybeast.com/newsweek/2009/12/07/work-the-new-digital-sweatshops.html

17. Zogaj, S., Bretschneider, U., \& Leimeister, J. M. (2013). Crowdtesting with testCloud - Managing the Challenges of a Crowdsourcing Intermediary: University of St. Gallen, The Case Centre. Available at: http://www.thecasecentre.org/educators/products /view?id= 117398 